\title{
Dislocation mediated alignment during metal nanoparticle coalescence
}

A.P. Lange ${ }^{\mathrm{a}, \mathrm{b}}$, A. Samanta ${ }^{\mathrm{a},{ }^{*}, \text { H. Majidi }}{ }^{\mathrm{b}}$, S. Mahajan ${ }^{\mathrm{b}}$, J. Ging ${ }^{\mathrm{a}}$, T.Y. Olson ${ }^{\mathrm{a}}$, K. van Benthem ${ }^{\mathrm{b}}$, S. Elhadj $\mathrm{j}^{\mathrm{a}, *}$

*samanta1@1lnl.gov

**elhadj2@1lnl.gov

${ }^{\text {a }}$ Lawrence Livermore National Laboratory

${ }^{\mathrm{b}}$ Department of Materials Science and Engineering, University of California, Davis

\section{Keywords}

Nanoparticle coalescence; nanoparticle sintering; dislocations; twinning; oriented attachment

\begin{abstract}
Dislocation mediated alignment processes during gold nanoparticle coalescence were studied at low and high temperatures using molecular dynamics simulations and transmission electron microscopy. Particles underwent rigid body rotations immediately following attachment in both low temperature $(500 \mathrm{~K})$ simulated coalescence events and low temperature $(\sim 315 \mathrm{~K})$ transmission electron microscopy beam heating experiments. In many low temperature simulations, some degree of misorientation between particles remained after rigid body rotations, which was accommodated by grain boundary dislocation nodes. These dislocations were either sessile and remained at the interface for the duration of the simulation or dissociated and crossslipped through the adjacent particles, leading to improved co-alignment. Minimal rigid body rotations were observed during or after attachment in high temperature $(1100 \mathrm{~K})$ simulations, which is attributed to enhanced interfacial diffusion. However, particle rotation was eventually induced by $\{111\}$ slip on planes parallel to the neck groove. These deformation modes led to the formation of single and multi-fold twins whose structures depended on the initial orientation of the particles. The driving force for $\{111\}$ slip is attributed to high surface stresses near the intersection of low energy $\{111\}$ facets in the neck region. The details of this twinning process were examined in detail using simulated trajectories, and the results reveal possible mechanisms for the nucleation and propagation of Shockley partials on consecutive planes. Deformation twinning was also observed in-situ using transmission electron microscopy, which resulted in the co-alignment of a set of the particles' $\{111\}$ planes across their grain boundary and an increase in their dihedral angle. This constitutes the first detailed experimental observation of deformation twinning during nanoparticle coalescence, validating simulation results presented here and elsewhere.
\end{abstract}

\section{Introduction}

Solid-state coalescence occurs when two initially isolated particles bond together in order to reduce their surface energies. Coalescence governs the initial stage of sintering when particles have translational and rotation degrees of freedom. It also occurs during crystal growth in solution when small nuclei attach to form larger nuclei [1-4]. Conventional models for 
coalescence were developed in the context of sintering and describe densification processes by diffusive transport mechanisms (e.g. surface diffusion, grain boundary diffusion, and evaporation/condensation) [5-7]. These models may be used to describe neck growth, but, with the exception of grain boundary diffusion, fail to describe processes by which particles align their crystallographic axes.

Particle alignment processes appear to play an important role during the coalescence of smaller particles $(\sim 1-10 \mathrm{~nm})$, for which interfaces and surfaces can greatly influence particle-particle interactions. The thermodynamics of these processes are relatively straightforward - alignment reduces the total energy of the system by removing or reducing strain at the particles' interface associated with a grain boundary. A number of groups observed the rotation of nanoparticles prior to or during attachment using in-situ liquid-phase and in-vacuo TEM [1-3,8-10]. These rotations corrected misorientation and, in some cases, led to perfectly aligned, coherent particles. The driving force(s) for this process, commonly referred to as oriented attachment (OA), are not well understood. Furthermore, the conditions that yield particle alignment or lead to extended defects within the agglomerated crystal have not been realized due to the complexity of this process. Li et al. suggested long range solvation and van der Waals forces may be involved in OA when coalescence occurs in solution [2]. Imperfect OA was observed by Penn and Banfield [11], Li et al. [2], and others, during which particles did not fully correct misalignment during $\mathrm{OA}$ and the resulting interface consisted of grain boundary dislocations. It was suggested that subsequent grain boundary migration or dislocation motion could lead to further co-alignment, however the dynamics of these processes, if they occur, remain unclear. Aabdin et al. investigated OA in gold nanoparticles using molecular dynamics (MD) and identified a critical tilt angle $\left(\sim 15^{\circ}\right)$ above which particles did not fully correct misalignment when they had common, adjoining (111) planes [3]. This result suggested that under prescribed initial conditions, sessile dislocations may form at interfaces during coalescence and inhibit further coalignment. However, the stability of such defects (whether or not they are sessile) and their role in subsequent co-alignment have not been rigorously examined by MD or experiment.

Twins were also observed after coalescence during in-situ TEM experiments involving nanoparticles of iron oxyhydroxide [2], palladium [9], and gold [12]. These twins were often ascribed to preferential particle attachment when the misorientation between particles was consistent with a low energy grain boundary. In MD simulations, however, twins were seen to occur by $\{111\}$ slip. This raises the question of whether twins form by OA or deformation processes during coalescence. Zhu and Averback, using MD, first observed the deformation of $\{111\}$ planes parallel to the neck groove during the coalescence of aligned and misoriented $\sim 5$ $\mathrm{nm}$ copper particles at $700 \mathrm{~K}$ [13]. They attributed this deformation to a high surface stress in the neck groove which arises from an imbalance of surface tension at the intersection of the particle surfaces (a dihedral angle smaller than the equilibrium value). Similar deformation behavior involving $\{111\}$ slip systems parallel to the neck groove was observed during MD studies of aluminum [14], palladium [15], and nickel [16] . Most early MD simulations of nanoparticle coalescence were run for short times ( 100 picoseconds) until neck growth terminated. However, coalescence simulations run for longer times ( $\sim 5$ nanoseconds) revealed that these deformation modes lead to twins well after the neck is formed. Cheng and Ngan studied these twin structures 
by simulating the coalescence of particles in the range of 2 to $4 \mathrm{~nm} \mathrm{[17].} \mathrm{They} \mathrm{found} \mathrm{that}$ twinning occurred predominantly by the slip of Shockley partials on consecutive planes. However, the driving force for the observed twinning remains unclear. Furthermore, many details of the twinning mechanism are not well understood such as the source of partial dislocations which glide on parallel $\{111\}$ planes, the connection between the initial misorientation and the final twin structure, and the high stability of the resulting coalesced, twinned structures. More significantly, no experimental observations of deformation twinning during nanoparticle coalescence have been made to support these in-silico results.

In this study, the dynamics of nanoparticle coalescence were systematically studied in-vacuo under conditions where dissolution, recrystallization, and solvation effects were eliminated. Particle alignment processes and deformation twinning during the coalescence of gold nanoparticles were identified at low and high temperatures using MD simulations and corroborated by in situ TEM heating experiments. Dissociation and glide of grain boundary dislocations after imperfect OA was observed during low temperature simulations, which led to additional co-alignment between particles. Deformation twinning was seen in all high temperature simulations in which the particles had common (100) faces. It was not observed, however, when the particles had common (111) faces. Particle rotation following neck formation was observed during in-situ TEM beam exposure experiments, consistent with the simulation results. Deformation twinning was also observed during in-situ beam exposure TEM experiments without heating, which resulted in the alignment of the coalesced particles' $\{111\}$ planes and an increase in their dihedral angle. A model is proposed for the coalescence of faceted particles, which considers the energy cost associated with neck growth. By using a combined simulation and experimental approach, microscopic insights into the dynamics of coalescence were developed which provide a framework for the development of processing strategies for nanocrystalline materials where the formation of metastable defects may limit or enhance their physical properties.

\section{Molecular dynamics simulations}

\subsection{Methodology}

Coalescence events of $5 \mathrm{~nm}$ gold nanoparticles with multiple misorientation angles were simulated at 500 and $1100 \mathrm{~K}$. Misorientation is denoted using common grain boundary notation for which rotation angle, rotation axis, and interfacial plane are specified (e.g. $10^{\circ}[010](100)$ describes a $10^{\circ}$ rotation of one particle about the [010] axis which was initially aligned with its (100) face coincident with the other particle's (100) face). Tilt misorientations of $10^{\circ}[010](100)$, $20^{\circ}[010](100)$, and $40^{\circ}[010](100)$, twist misorientations of $45^{\circ}[100](100), 45^{\circ}[111](111)$, and initially aligned particles, $0^{\circ}(100)$ and $0^{\circ}(111)$, were analyzed. Prior to simulating coalescence, the particles were thermalized separately by increasing their temperatures at a rate of 4 $\mathrm{K} /$ picosecond from room temperature, then annealing at $500 \mathrm{~K}$ or $1100 \mathrm{~K}$ for $50 \mathrm{ps}$. Coalescence was simulated for $5 \mathrm{~ns}$ with $1 \mathrm{fs}$ time steps under the constant temperature (NVT) ensemble using the Large-scale Atomic/Molecular Massively Parallel Simulator (LAMMPS). A Nose- 
Hoover thermostat was used with a 100 time step damping parameter. Interatomic interactions were modeled using an embedded atom model type potential developed by Auckland et al. [18]. The potential was fit to empirical data for the equilibrium f.c.c. lattice parameter, cohesive energy, $\mathrm{C}_{11}, \mathrm{C}_{12}$, and $\mathrm{C}_{44}$ elastic constant, the vacancy formation energy, and the stacking fault energy. The cutoff radius for pairwise energy calculations was set to $5 \AA$, which corresponds to about 1.73 times the separation of nearest neighbors in the equilibrium volume f.c.c. lattice. The potential decays smoothly to zero at the cutoff value. To analyze the microscopic mechanisms, snapshots saved during the coalescence simulations were quenched using a 1000 step steepest decent minimization scheme. Analysis of MD trajectories and dislocations were carried out using OVITO [19] and the dislocation extraction algorithm (DXA) [20], respectively. DXA identifies arbitrary dislocations by integrating over discrete Burgers circuits around displaced regions of the crystal. Although it was used to gain a qualitative understanding of dislocation behavior during coalescence, specific Burgers vectors described in the text were identified by analyzing atomic displacements unless noted otherwise.

\subsection{Rotation during and immediately following attachment (oriented attachment)}

At low temperatures ( $500 \mathrm{~K}$, or $\sim 0.37 T_{\mathrm{m}}$ where $T_{\mathrm{m}}$ is the melting point), particles in-vacuo with tilt misorientations underwent rigid body rotations during attachment (Fig. 1). Rotation during attachment was minimal at high temperatures $\left(1100 \mathrm{~K}\right.$, or $\left.\sim 0.82 T_{\mathrm{m}}\right)$. A comparison of the dynamic attachment processes for coalescence events with $10^{\circ}[010](100)$ initial misorientations at $500 \mathrm{~K}$ and $1100 \mathrm{~K}$ is provided in Fig. 1. Figures 1(a) and (c) show plots of the angular separation between the [100] crystallographic axes of two coalescing particles $(\Delta \theta[100])$ versus time at $500 \mathrm{~K}$ and $1100 \mathrm{~K}$, respectively. The images labeled (i-iii) and (iv-vi) show atomic structures of the particles viewed along the [010] axis at various stages of attachment as labeled in Figs. 1(a) and (c). Figure 1(b) shows a plot of $\Delta \theta[100]$ versus time for the first $25 \mathrm{ps}$ of attachment during $10^{\circ}[010](100)$ and $20^{\circ}[010](100)$ coalescence events at $500 \mathrm{~K}$. Movies of the particle trajectories for events corresponding to Figs. 1(a)-(c) are provided in the supplementary section (SV1-SV3, respectively).

During the $500 \mathrm{~K}, 10^{\circ}[010](100)$ simulation, neck growth terminated after $\sim 10 \mathrm{ps}$, as indicated by label (i) in Fig. 1(a) and shown in structure (i). Very little rotation $\left(<1^{\circ}\right)$ occurred before or during neck growth. It is worth noting that $\mathrm{Li}$ et al. observed extensive particle rotation prior to neck formation during nanoparticle coalescence in solution [2], so this observation may be unique to in-vacuo systems. After neck growth terminated, the particles rotated until their crystallographic axes were aligned (point (ii)). The particles continued to rotate past $\Delta \theta[100]=0$, and a region near the interface plastically deformed (label (iii)), presumably because the crystal was strained beyond its elastic limit. The resulting dislocations were sessile and remained at the interface, along with the misorientation, for the duration of the simulation ( $5 \mathrm{~ns}$ ). Due to residual angular momentum in the system, the particles continued to oscillate elastically, as indicated between 100 and 200 ps in Fig. 1(a).

The comparison between the $\Delta \theta[100]$ versus time plots for the $10^{\circ}[010](100)$ and $20^{\circ}[010](100)$ simulations at $500 \mathrm{~K}$ in Fig. 1(b) shows that the particles rotated with nearly identical angular velocities during the first $25 \mathrm{ps}$. This suggests that the torque acting on the particles immediately 
following neck formation was comparable in the two simulations; a surprising result as there were significant differences in the angular relationship between adjoining surface facets and the degree of elastic strain at the interface (determined by visual inspection of the trajectories). Rigid body rotations during attachment at $500 \mathrm{~K}$ were highly dynamic and depended on localized perturbations caused by fluctuations of surface atoms on adjoining facets. Simulations run with similar initial atomic positions but different randomly assigned velocities did not produce the same oriented attachment behavior, which made it difficult to predict if a prescribed initial misorientation would lead to particle alignment.

Particles did not undergo rigid body rotations during attachment in the $1100 \mathrm{~K}$ simulations. This is highlighted by the $\Delta \theta[100]$ versus time plot of an event with a $10^{\circ}[010](100)$ misorientation in Fig. 1(c). After neck formation (point (j) in Fig. 1(c)), the particles retained their $10^{\circ}[010](100)$ misorientation, as shown by structure (j). At higher temperatures, diffusive atomic motion at the interface may relieve torque before particle rotation can occur. After attachment, slip occurred in both particles which led to plastic deformation along $\{111\}$ planes parallel to the neck groove (structure jj) and an increase in $\Delta \theta[100]$. Slip then occurred on a common set of parallel $\{111\}$ planes across the grain boundary (structure jjj) which improved co-alignment. Deformation continued after a coherent, defect free interface was observed which led to the formation of an 8 layer twin (not shown). Similar twinning events will be described in detail in the "deformation twinning" section.

\section{Fig. 1}

\subsection{Defect mediated co-alignment after imperfect $O A$}

In the $500 \mathrm{~K}$ simulations for which misorientation was not fully corrected during OA, the interface consisted of strained regions accommodated by dislocations. An example of this is shown in Fig. 2(a). During this simulation, in which the particles had an initial $45^{\circ}[100](100)$ initial misorientation but underwent rigid body rotations during attachment, a stable dislocation node was formed at the interface. This node remained at the interface, along with the misorientation, for $\sim 4$ ns. As shown in Figs. 2(b-e), the node dissociated after $\sim 4$ ns and glided through the adjacent particles which removed the associated strain and led to additional coalignment. Atoms in this figure are displayed according to their coordination - atoms with fcc coordination are colored green, atoms with hcp coordination are colored red, and atoms with unidentified coordination are colored white. They are shown with 50\% transparency to allow for visualization of processes below the surface.

The node circled in Fig. 2(a), contained a sessile Lomer-Cotrell lock with a stair-rod dislocation, displayed in red, and trailing Shockley partials, labeled 1 and 2. It also contained a perfect $1 / 2[\overline{1} 0 \overline{1}]$ dislocation, displayed in yellow. The Burgers vectors of the trailing partials, $1 / 6[112]_{(\overline{11} 1)}$ and $1 / 6[1 \overline{2} 1]_{(\overline{111})}$, were determined by examining the displacements of stacking faults between the leading and trailing partials. The Burgers vector of the perfect dislocation (displayed in yellow) was determined by examining the atomic displacements of the dissociated partials, $1 / 6[\overline{112}]_{(\overline{11} 1)}$ and $1 / 6[\overline{2} 1 \overline{1}]_{(\overline{11} 1)}$, near the surface. It was assumed that the sum of the 
Burgers vectors at the node was zero which implied that the red dislocation was a stair-rod dislocation with a Burgers vector of 1/6[110].

The stair-rod dislocation dissociated after $\sim 4 \mathrm{~ns}$, as shown in Fig. 2(b). One of the partials then detached from the node, shown in Fig. 2(c), and the partials labeled a, b, and c reacted to form a single stacking fault bounded by two Shockley partials (Fig. 2(d)). These partials then reacted to form a perfect, $1 / 2<110\rangle$ dislocation which glided to the surface and left the particles aligned (Fig. 2(e)).

\section{Fig. 2}

In many simulations at $500 \mathrm{~K}$ with initial tilt misorientations, interfacial dislocations after imperfect OA were sessile and remained at the interface for the duration of the simulation (5 ns). When the misorientation between particles was of pure twist type after attachment, strain at the interface was confined to two interfacial layers. These layers consisted of sheared domains that were coincident with the adjacent particles. The atomic structure of strained, interfacial layers for a $45^{\circ}[100](100)$ coalescence event are shown in Fig. 3. This figure shows atoms associated with three (100) layers at the interface, viewed along the [100] direction. The red and yellow atoms were initially coincident with the particle behind the plane of the page and the blue atoms were coincident with the particle above the plane of the page, as shown by the $45^{\circ}$ angle between them in the top left image. After a neck was formed, the yellow and blue atoms, which lied on top of the red atoms in the perspective of the figure, consisted of sheared domains which accommodated strain between the two particles. As the particles became more aligned, a greater number of atoms in the adjacent particles overlapped according to the coincident site lattice and it was only between these coincident domains that shearing occurred. This is illustrated in the bottom four images of Fig. 3 where the coincident regions are outlined with blue rectangles. The coincident domains grew as the particles rotated, and the slip lines moved toward the surface until the interface was coherent as shown in the bottom right image of Fig. 3.

\section{Fig. 3}

\subsection{Deformation twinning}

Deformation twinning was active during all coalescence simulations at $1100 \mathrm{~K}$ in which the particles had common, adjoining (100) faces. In fact, twinning was even observed when the particles were initially aligned (when the adjoining atomic planes were in perfect registry). This case is particularly interesting as it highlights the importance of the evolving surface stress in the neck groove (discussed in detail below). Although the twins formed by the glide of Shockley partials on consecutive planes, the details of this process remain unclear. In particular, the associated dislocations reactions which led to the formation of Shockley partials and the role of perfect dislocations were not resolved. However, dislocation nucleation events, activated slip planes, and final twin structures for varied initial misorientations were examined and will be utilized propose possible mechanisms. The simulation results presented below were all carried out at $1100 \mathrm{~K}$. 
Particles that were initially aligned with common (100) faces $\left(0^{\circ}(100)\right)$, had a coherent interface after neck formation as shown in Fig. 4(a). The atoms in Fig. 4 are colored according to their coordination, displayed with 50\% transparency, and viewed along their [011] crystallographic axis. After $\sim 26$ ps, dislocations nucleated from surface shearing events and cross-slipped onto a (11̄1) plane parallel to the neck groove. After this initial shearing event, a full activation of the (1111) slip system occurred and dislocations were seen to rapidly nucleate or cross-slip and glide on consecutive planes. This led to many stacking faults on parallel planes, as shown in Fig. 4(b). Although a twin nucleus was generated early on during this process (Fig. 4(b)), it did not propagate immediately but was removed by further glide of Shockley partials. The $(\overline{11} 1)$ slip system then became active in the right hand particle as shown in Fig. 4(c) which eventually led to the formation of the two-fold twin in Fig. 4(d). Deformation terminated after the twins formed which suggests that the driving force for dislocation nucleation and glide was relaxed.

\section{Fig. 4}

Dislocations which led to the initial (1 $\overline{1} 1)$ slip event in the $0^{\circ}(100)$ coalescence simulation were nucleated from surface shearing events, as shown in Fig. 5. Again, the atoms in Fig. 5 are colored according to their coordination, however they are not displayed with transparency. Multiple (111) planes are shown which penetrated the interfacial region between the particles. In Fig. 5(b), the surface atoms, enclosed by dotted lines, were sheared by a Shockley partial, $1 / 6<112>$, which led to the formation of a stacking fault between the surface and underlying layer (the details of this event are presented with a different perspective in the supplementary figure, S1). One atom in the stacking fault can be seen (appears red due to hcp coordination) below the sheared region in Fig. 4(b). The next frame, Fig. 4(c), shows a 1/6[121] Shockley partial that glided into the interface on a $(\overline{1} 11)$ plane and terminated on the boundary of the stacking fault. This dislocation glided further into the interface, as shown in Fig. 4(d), then glided back to the surface. Similar nucleation events occurred during the first $\sim 26 \mathrm{ps}$ of the simulation which produced perfect dislocations, $1 / 2\langle 110\rangle$, Hirth dislocations, $1 / 3\langle 100\rangle$, and Shokley partials, as identified by the DXA algorithm. After $\sim 26 \mathrm{ps,} \mathrm{one} \mathrm{dislocation} \mathrm{node} \mathrm{reacted}$ and cross-slipped onto a (1111) plane parallel to the neck groove which activated the (1111) slip system as discussed above.

\section{Fig. 5}

The dislocation nucleation mechanisms, activated slip systems, and final twin structures differed for coalescence events with common $\{100\}$ planes but different initial misorientations. In systems with a high degree of misorientation, dislocations were seen to nucleate predominantly at disordered grain boundaries, as shown in Fig. 6(a) (atoms colored according to coordination without transparency). These dislocations (primarily Shockley partials) nucleated and disappeared readily, leaving the particles unchanged and preserving their relative orientations. Similar to the case of initially aligned particles, in a $45^{\circ}[100](100)$ simulation, one dislocation nucleated at the grain boundary and cross slipped onto a (1111) plane after $280 \mathrm{ps,} \mathrm{which} \mathrm{led} \mathrm{to}$ the full activation of slip on parallel planes. 


\section{Fig. 6}

When the particles had small initial tilt misorientations, the activation of $\{111\}$ slip parallel to the neck groove occurred by the dissociation and cross-slip of low-angle grain boundary dislocations. An example of this is shown in Fig. 6(b) for the $10^{\circ}$ [010](100) event discussed in Fig. 1 (atoms are colored according to coordination with transparency). Figure 6(b) shows the dissociation of a grain boundary dislocation into a sessile Hirth lock and two Shockley partials,

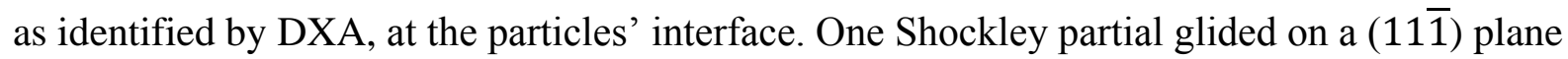
(bottom right image of Fig. 6(b)). The misorientation of the particles was then removed by the activation of both the $(11 \overline{1})$ and $(1 \overline{1} 1)+\delta$ slip planes, where $\delta$ represents the rotation of the right hand particle relative to the left hand particle, as shown in the supplementary figure, S2a.

The final, stable structures for six coalescence events at $1100 \mathrm{~K}$ with different misorientations are shown in Fig. 7 with the same coloring scheme as Fig. 4. Fast Fourier transforms (FFTs) of the images are also shown. All structures contained twins except the one corresponding to a $45^{\circ}[111](111)$ twist misorientation. Initially aligned particles with common (111) faces formed three layer twins parallel to their interface. This acted to stabilize the neck groove by forming a $\{111\}$ plane at the center of the groove (shown in the supplementary figure, S3). This twin appeared to form by the diffusion of interfacial atoms, not by deformation, as was the case in simulations with common (100) faces. The single crystal, coalesced structure from the $45^{\circ}[111](111)$ simulation, and the varied single and two-fold twin configurations in Fig. 7 suggest it may be possible to tailor twinning by controlling misorientation.

\section{Fig. 7}

\section{Transmission electron microscopy}

\subsection{Methodology}

Alignment processes during coalescence were explored experimentally using conditions analogous to those used in the simulations presented above. Gold nanoparticle coalescence was induced by light electron beam heating to replicate low temperature conditions, while high temperature conditions were produced by resistive heating at $\sim 1073 \mathrm{~K}$ for $\sim 20$ minutes with a Protochips holder and amorphous carbon coated silicon carbide heater chips. A JEOL 2500 SE microscope operated at $200 \mathrm{kV}$ was used for all annealing experiments. The temperature increase of an amorphous carbon film under e-beam irradiation with an accelerating voltage of $80 \mathrm{kV}$ and a current density of $5000 \mathrm{e} \cdot \AA^{-2} \mathrm{~s}^{-1}$ was previously estimated to be $\sim 30 \mathrm{~K}$ [21]. The ensemble energy loss of electrons through a sample at $200 \mathrm{kV}$ is roughly half that at $80 \mathrm{kV}$ due to a larger mean free scattering path. Therefore, it is expected that the temperature increase during beam heating experiments was less than $\sim 15 \mathrm{~K}$. The interaction of metal nanoparticles under the electron beam is complicated by the generation of plasmons which give rise to long range electrostatic interactions between particles and between particles and the electron beam [22]. Furthermore, interaction between the gold nanoparticles and the underlying carbon film may have restrained their translational and rotational movements. Therefore, particle motion prior to attachment is not addressed here, although similar inter-particle displacements and interactions 
were reported for solvent and vacuum conditions [2-4,23]. Instead, focus was placed on interactions following particle contact that led to improved co-alignment and deformation.

Particles were inspected after annealing for the high temperature coalescence experiment. Imaging was not carried out before or during coalescence to minimize any disturbance from the electron beam. Additional images of the particles were acquired using a JEOL 2100 aberration corrected scanning transmission electron microscope (STEM) operated at $200 \mathrm{kV}$ with a high angle annular dark field detector after removing the holder from the TEM vacuum chamber. Two sets of gold nanoparticles were used. One set, prepared in house, was used for the low temperature, beam heating experiments. The other, prepared by BBI Solutions, was used for the high temperature experiment. Both were grown by the citrate reduction of a gold chloride solution. It was found that the batch prepared by BBI was mono-disperse with particle diameters equal to $\sim 5 \mathrm{~nm}$. A large percentage of the particles contained five-fold twins. The batch prepared in-house consisted of particles in the range of 3-10 nm which were mostly single crystalline. Both sets of particles were terminated with a carboxyl layer. The influence of such capping layers was previously investigated [24]. Their role is uncertain in the context of dislocation mediated processes after attachment as they may influence surface energies and diffusive processes in the neck groove.

\subsection{Particle rotation after attachment}

Figs. 8(a)-(c) show particle rotation after attachment during electron beam exposure $(\mathrm{T} \leq 315 \mathrm{~K})$. The two particles were initially misaligned when they came into contact. This is apparent from Fig. 8(a) in which lattice fringes can be seen in the bottom particle near the interface but not in the top particle. The lack of lattice fringes in the top particle indicates that it was rotated away from a zone axis. Between the first frame, shown in Fig. 8(a), and the second (Fig. 8(b)), the smaller particle rotated while the larger particle did not. Both particles then rotated and an increase in the neck width was observed, as indicated by the dark black lines in Fig. 8(c). This last rotation resulted in the near-perfect co-alignment of the particles' $\{111\}$ planes (planes identified by fringe spacing) as indicated by the white, dashed lines, however a slight misorientation remained between the particles.

\section{Fig. 8}

Another example of particle rotation after attachment is shown in Figs. 8(d) and (e). The spacing of the lattice fringes are $2.31 \pm 0.09 \AA$ ( \pm values correspond to the standard deviation of multiple measurements) and $2.31 \pm 0.05 \AA$ for the left hand and right hand particles, respectively. This is consistent with the $\{111\}$ interplanar spacing for gold, $2.35 \AA$. The particle on the right had clear facets with 6-fold symmetry, as indicated by the white dashed line in Fig. 8(d) and the red dashed line in Fig. 8(e). The particles were in contact when the image in Fig. 8(d) was acquired. The particle on the right then underwent a $22^{\circ}$ rotation as indicated by the change in angle of its surface facets and FFT spots in Fig. 8(e). Both particles then underwent additional rotation toward zone axes, which allowed their crystallographic axes to be indexed. Using this information, the plane parallel to the interface in the right hand particle after the $22^{\circ}$ rotation was 
determined to be $\{110\}$. This does not correspond to a misorientation examined using molecular dynamics in this study.

\subsection{Twinning during beam exposure experiments}

Figure 9(a) shows the particles in Fig. 8(e) after they rotated toward their [110] and [112] zone axes, respectively. The particle on the left subsequently underwent deformation. A region of the particle, outlined by the white triangle in Fig. 9(b), initially became dark which indicates the atomic columns in this area were disordered. The area of this disordered region grew, then the atomic columns became visible again, shown in Fig. 9(c). It can be seen by comparing the $\{111\}$ lattice fringes in the left and right particles in Fig.s 9(a) and (b) (traced with white dashed lines) that these $\{111\}$ planes were initially misaligned. However, the $\{111\}$ planes in the deformed region of the particle on the left were aligned with the $\{111\}$ planes in the particle on the right (Fig. 9(c)). Furthermore, the planes that did not intersect the interface were not deformed, highlighted by the fringes outside of the triangular region. The high resolution, selected area inset and FFT in Fig. 9(d) show that a twin formed at the boundary of the deformed and undeformed regions. The twin boundary was not perfect, however, but consisted of Shockley partials, as highlighted in the selected area inset. The twin penetrated the neck groove and resulted in an increase in the dihedral angle between the planes, traced by the red dashed lines in Fig.s 9(b) and (c).

\section{Fig. 9}

\subsection{Twinned structures after high temperature annealing}

The twin structures in particles that coalesced at high temperatures ( 1073 K) showed many similarities to the stable structures observed in MD. The majority of the un-coalesced, asreceived five-fold twinned particles retained their five-fold twin structures even after annealing at $1073 \mathrm{~K}$ for 20 minutes. An example is shown in Figs.10(a) and (b). The TEM image in Figure 10(a) shows a particle on a zone axis prior to annealing and the STEM image in Fig. 10(b) shows a particle near a zone axis after annealing. Three examples of coalesced particles after high temperature annealing are shown in Figs. 10(c)-(e). The structure in Fig. 10(c) was mostly single crystalline but had a dark region (upper left corner) which was not on a zone axis. This structure had large $\{111\}$ facets and stepped non- $\{111\}$ surfaces shown by the dotted line and arrow in Fig. 10(c), respectively. The structure in Fig. 7 for the $45^{\circ}[111](111)$ simulation was very similar. Approximately half of the 2-particle agglomerates were not on a zone axis but had aligned lattice fringes with no visible defects (not shown). Figs. 10(c) and (d) had twin boundaries as indicated by the arrow and dashed line, respectively. The angle between the (002) and twinned (111) FFT spots in the inset of Fig. 10(c) is $\sim 14^{\circ}$ which matches the value obtained using the FFT spots in Fig. $7\left(\sim 16^{\circ}\right)$. The twin in Fig. 10(d) is difficult to see, however the angular separation of the spots $\left(\sim 16^{\circ}\right)$ is discernable in the selected area FFT.

\section{Fig. 10}

\section{Discussion}




\subsection{Thermodynamics of small particle coalescence}

The thermodynamic driving force for coalescence is a reduction in surface energy. Particle surfaces have high free energies relative to the bulk due to un-coordinated bonds. By bonding to each other, particles are able to reduce their surface areas and thereby reduce their free energies. The change in Gibbs free energy associated with this reduction in area can be written,

$$
\Delta G=\int d \gamma d A=\gamma \Delta A+A \Delta \gamma,
$$

where $\gamma$ is the surface energy and A is the surface area. After particles form a small neck (share some bonds on their surfaces), diffusive processes have been shown to dominate neck growth in larger particle systems at high temperatures. In classical models for coalescence and sintering, depicted in Fig. 11(a), high curvature in the neck groove is assumed to give rise to a chemical potential gradient as given by the Gibbs-Thomson relation. Surface energy in these models is either assumed to be constant or to increase with curvature (smaller radius of curvature, r, in Fig. 11(a)). Therefore, the second term in the equation above is either zero or negative. The first term is negative because the particles reduce their total area during neck growth. This implies that neck growth occurs spontaneously.

\section{Fig. 11}

Smaller particles have greater surface area to volume ratios. This implies that the driving force for a small particle to maximize its low energy facets will greater. This has an interesting consequence for the coalescence of nanoparticles. After two faces bond during neck formation, if the adjacent surfaces that form the neck groove are low energy facets, the system can become kinetically trapped as the energy required to remove the low energy facets in favor of a higher energy surface is substantial. This kinetic barrier can be understood using the schematic in Fig. 11(b). If two low energy facets, $S_{1}$ and $S_{2}$, associated with two particles of radius a, intersect with a dihedral angle, $\theta$, the change in Gibbs free energy associated with creating a new surface, $S_{3}$, in order to grow the neck is

$$
\Delta G=\gamma_{1} \Delta S_{1}+\gamma_{2} \Delta S_{2}+\gamma_{3} \Delta S_{3},
$$

where $\Delta S_{i}$ is the change in surface area and $\gamma_{i}$ is the surface energy of facet $i$. If it is assumed that the created surface forms the same angle with both of the original facets, the expression above becomes

$$
\Delta G=\Delta S_{3}\left[\gamma_{3}-1 / 2\left(\gamma_{1}+\gamma_{2}\right) \sin \theta / 2\right] .
$$

Using this expression, it can be determined whether neck growth will be spontaneous for a given set of surfaces intersecting at prescribed angles. This is easily done in the case of initially aligned particles with common (100) faces discussed above. Two planes that intersect in the neck groove are $(1 \overline{1} 1)$ and $(\overline{11} 1)$. The angle between these planes is $\sim 131.81^{\circ}$ and the plane normal to the neck groove is $(0 \overline{1} 1)$. Using surface energies of 1.137 and $1.414 \mathrm{~J} / \mathrm{m}^{2}$ for $\{111\}$ and $\{110\}$ planes, respectively [25], it is found 


$$
\frac{\Delta G}{\Delta S_{3}}=\gamma_{\{110\}}-\gamma_{\{111\}} \sin \theta / 2 \approx 0.38 \mathrm{~J} / m^{2}>0
$$

Therefore, there exists a barrier for a $\{110\}$ face to form along this line of intersection and neck growth does not occur spontaneously. Despite this barrier, large surface stresses exist where these facets meet due to an imbalance of surface tension which can give rise to a resolved shear stress on parallel planes (as pointed out by Zhu and Averback [13]).

\subsection{Oriented attachment}

MD simulations showed that rigid body rotations occur at low temperatures immediately following attachment. This differs from observations of OA in solvent conditions in which particle rotation was observed prior to attachment. Rotations immediately following attachment were found to be highly dynamic and sensitive to small perturbations on the surfaces of intersecting faces. This suggests that any phenomenological model for OA based on prescribed initial configurations may be impractical for in-vacuo systems. Rigid body rotations during or following attachment were not observed in high temperature simulations. This is attributed to enhanced diffusion of atoms at the interface which responded to the strain environment before rotations could occur. Therefore, there should be a temperature above which OA does not occur for a given system that depends on the constituent species of the particles and their bulk and surface mobilities.

\subsection{Defect mediated rotation}

Dislocation nodes were observed after imperfect OA at the interface of coalesced particles with tilt or tilt and twist misorientations during low temperature MD simulations. These dislocations were either sessile or dissociated and glided through the adjacent particles on $\{111\}$ slip planes. This process can be understood in the context of the model above. If neck growth is inhibited by the stability of intersecting $\{111\}$ facets, a high surface stress will exist at the intersection of these planes due to an imbalance in surface tension. Dislocations at the interface are then subject to a high resolved shear stress on $\{111\}$ planes parallel to the neck groove and may dissociate and glide through the particles to remove misalignment.

The resolved shear stress on $\{111\}$ planes depends on the surface tension in the neck groove, and therefore the angles between intersecting surface facets. It is expected that dislocation mediated alignment after imperfect OA will occur more readily for certain misorientations (e.g. those with common (100) faces). However, because OA is dynamic at low temperatures, predicting the resulting crystallography of the neck groove based on initial configurations may prove difficult.

\subsection{Deformation twinning during coalescence}

Deformation twinning was observed during beam exposure TEM experiments. This process was similar to what was seen during high temperature simulations in which partial dislocations nucleated at high angle grain boundaries and glided on slip planes parallel to the neck groove. The twins in both the simulated structures shown in Fig. 7 and the coalesced particles in Fig. 9 led to a reduced dihedral angle and improved co-alignment along the grain boundary. The 
activation of this process at low temperatures during beam exposure may be attributed to the time-scale limitations of MD. If deformation twinning is thermally activated by the nucleation of partial dislocations on the surface of the neck groove or at grain boundaries, this process will only occur at high temperatures over short time intervals ( $\leq 5 \mathrm{~ns})$. However, the beam exposure experiment in Fig. 7 was carried out over the course of hours. On this time scale, the activation barrier for dislocation nucleation may be overcome. Other groups observed twin structures after metal nanoparticle coalescence similar to those seen in the TEM experiments presented here [15].

According to the analysis above, kinetic barriers to neck growth may only form in regions of the neck groove where low energy facets intersect with small dihedral angles. This will give rise to slip on planes parallel to these facets. MD simulations revealed that multiple slip systems can become activated and deactivated prior to twin formation. As surface stress is relaxed between a set of facets by slip, the dihedral angle between other facets in the neck groove may decrease leading to the activation of other slip systems.

In the high temperature simulations, slip systems parallel to the neck groove were initiated by a single cross-slip event of dislocations nucleated near the interface. The rapid activation of the slip system after this initial event may be due to lower energetic barriers associated with shearing a crystal on parallel planes after an intrinsic stacking fault is formed. Glide of the initial partial produced a two layer stacking fault. If the crystal sheared again by partial slip on a plane adjacent to that of the initial slip event, the crystal would still only contain two faulted layers. Subsequently, partial slip could occur on a plane adjacent to one of the resulting faulted planes and the crystal would still only contain two faulted layers. In this way, consecutive layers may become easily sheared after the initial slip event as the total faulted area, and therefore the free energy of the system is not substantially increased.

Slip on $\{111\}$ planes also necessarily produced surface steps on intersecting $\{111\}$ facets in the neck groove. These steps produced a locally strained environment, which could have triggered the nucleation of partials on parallel planes by surface shearing events to restore low energy \{111\} surfaces (similar to what was observed in Fig. 5). This process, although not heavily substantiated by the results, would be consistent with a model in which deformation is driven by surface stresses in the neck groove. Once surface stresses are relaxed by deformation processes, nucleation and glide on parallel $\{111\}$ planes would terminate, as was observed after twin formation in the simulations. It is believed that the resulting twinned structures are not at a global thermodynamic minimum, but are very stable due to their additional $\{111\}$ surfaces and the associated reduction in dihedral angle. This is supported by the fact that many of the coalesced agglomerates appeared to be single crystalline after the high temperature TEM experiment. On longer time scales, the agglomerates may be able to overcome energy barriers associated with removing twins formed during coalescence.

\section{Conclusions}

Dislocation mediated alignment processes and deformation twinning during the coalescence of $\sim 5 \mathrm{~nm}$ gold nanoparticles were studied at low and high temperatures. Rigid body rotations were 
observed after attachment during low temperature simulations but not at high temperatures. Dislocations that arose from imperfect OA were either sessile or glided through adjacent particles. The driving force for this slip process was attributed to surface stress in the neck groove. Deformation twinning was observed at high temperatures during MD simulations and at low temperatures during beam heating TEM experiments. MD simulations revealed that this process is initiated by a single slip event on a $\{111\}$ plane parallel to the neck groove. The dislocations involved in this event nucleated from surface shearing events, low angle grain boundary dislocations, or near disordered high angle grain boundaries. The mechanisms responsible for slip on consecutive planes remain unclear, however it is proposed that partial nucleation from highly strained $\{111\}$ surfaces in the neck groove may be responsible.

Deformation twinning during coalescence under electron beam exposure led to the co-alignment of $\{111\}$ planes across the grain boundary of coalesced particles and an increase in their dihedral angle. The role of surface energy during neck growth was considered in the case of small, faceted particles and it was shown that a kinetic barrier exists when low energy facets intersect with small dihedral angles in the neck groove. This allows longer time-scale processes such as deformation twinning to occur which drive the system to stabilize its high energy surfaces.

\section{Acknowledgements}

This work was performed under the auspices of the U.S. Department of Energy by Lawrence Livermore National Laboratory under Contract DE-AC52-07NA27344. Additional funding was provided by the University of California, Davis College of Engineering.

\section{References}

[1] J.J. De Yoreo, P.U.P.A. Gilbert, N.A.J.M. Sommerdijk, R.L. Penn, S. Whitelam, D. Joester, H. Zhang, J.D. Rimer, A. Navrotsky, J.F. Banfield, A.F. Wallace, F.M. Michel, F.C. Meldrum, H. Colfen, P.M. Dove, Crystallization by particle attachment in synthetic, biogenic, and geologic environments, Science 349 (2015) aaa6760-aaa6760.

[2] D. Li, M.H. Nielsen, J.R.I. Lee, C. Frandsen, J.F. Banfield, J.J. De Yoreo, Directionspecific interactions control crystal growth by oriented attachment, Science. 336 (2012) 1014-1018.

[3] Z. Aabdin, J. Lu, X. Zhu, U. Anand, N.D. Loh, H. Su, U. Mirsaidov, Bonding pathways of gold nanocrystals in solution, Nano Lett. 14 (2014) 6639-6643.

[4] H-G. Liao, L. Cui, S. Whitelam, H. Zheng, Real-time imaging of Pt3Fe nanorod growth in solution, Science 336 (2012) 1011-4.

[5] B.J. Kellett, F.F. Lange, Thermodynamics of Densification: I, sintering of simple particle arrays, equilibrium configurations, pore stability, and shrinkage, J. Am. Ceram. Soc. 72 (1989) 725-734.

[6] F.F. Lange, B.J. Kellett, Thermodynamics of densification: II, grain growth in porous compacts and relation to densification, J. Am. Ceram. Soc. 72 (1989) 735-741.

[7] R. Castro, Sintering: Mechanisms of Convention Nanodensification and Field Assisted Processes, 1st ed., Springer, 2013. 
[8] Q. Chen, H. Cho, K. Manthiram, M. Yoshida, X. Ye, A.P. Alivisatos, Interaction Potentials of Anisotropic Nanocrystals from the Trajectory Sampling of Particle Motion using in Situ Liquid Phase Transmission Electron Microscopy, ACS Cent. Sci. 1 (2015) 33-39.

[9] B. Lim, H. Kobayashi, P.H.C. Camargo, L.F. Allard, J. Liu, Y. Xia, New insights into the growth mechanism and surface structure of palladium nanocrystals, Nano Res. 3 (2010) $180-188$.

[10] Y. Chen, R.E. Palmer, J.P. Wilcoxon, Electron beam induced sintering of passivated gold nanoparticles, Langmuir 22 (2006) 2851-2855.

[11] R.L. Penn, J.F. Banfield, Imperfect oriented attachment: dislocation generation in defectfree nanocrystals, Science 281 (1998) 969-971.

[12] A. Halder, N. Ravishankar, Ultrafine single-crystalline gold nanowire arrays by oriented attachment, Adv. Mater. 19 (2007) 1854-1858.

[13] H. Zhu, R.S. Averback, Sintering processes of two nanoparticles: a study by molecular dynamics simulations, Philos. Mag. Lett. 73 (1996) 27-33.

[14] J.S. Raut, R.B. Bhagat, K.A. Fichthord, Sintering of aluminum nanoparticles: a molecular dynamics study, Acta Metall. 10 (1998) 837-851.

[15] P. Grammatikopoulos, C. Cassidy, V. Singh, M. Sowwan, Coalescence-induced crystallisation wave in Pd nanoparticles, Sci. Rep. 4 (2014) 5779.

[16] I.N. Kar'kin, Y.N. Gornostyrev, L.E. Kar'kina, Molecular dynamics simulation of the formation of twin boundaries during agglomeration of nanoparticles, Phys. Solid State. 52 (2010) 431-435.

[17] B. Cheng, A.H.W. Ngan, The crystal structures of sintered copper nanoparticles: A molecular dynamics study, Int. J. Plast. 47 (2013) 65-79.

[18] G.J. Ackland, G. Tichy, V. Vitek, M.W. Finnis, Simple N-body potentials for the noble metals and nickel, Philos. Mag. A. 56 (1987) 735-756.

[19] A. Stukowski, Visualization and analysis of atomistic simulation data with OVITO-the Open Visualization Tool, Model. Simul. Mater. Sci. Eng. 18 (2010) 015012.

[20] A. Stukowski, V.V. Bulatov, A. Arsenlis, Automated identification and indexing of dislocations in crystal interfaces, Model. Simul. Mater. Sci. Eng. 20 (2012) 085007.

[21] H. Zheng, J.B. Rivest, T.A. Miller, B. Sadtler, A. Lindenberg, M.F. Toney, et al., Observation of transient structural-transformation dynamics in a $\mathrm{Cu} 2 \mathrm{~S}$ nanorod, Science 333 (2011) 206-209.

[22] P.E. Batson, A. Reyes-Coronado, R.G. Barrera, A. Rivacoba, P.M. Echenique, J. Aizpurua, Plasmonic nanobilliards: controlling nanoparticle movement using forces induced by swift electrons, Nano Lett. 11 (2011) 3388-3393.

[23] K.S. Cho, D. V. Talapin, W. Gaschler, C.B. Murray, Designing PbSe nanowires and nanorings through oriented attachment of nanoparticles, J. Am. Chem. Soc. 127 (2005) 
$7140-7147$.

[24] M.A. Asoro, D. Kovar, P.J. Ferreira, Effect of surface carbon coating on sintering of silver nanoparticles: in situ TEM observations., Chem. Commun. (Camb). 50 (2014) 4835-4838.

[25] J. Wang, S-Q. Wang, Surface energy and work function of fcc and bec crystals : density functional study, Surf. Sci. 630 (2014) 216-224. 
Dislocation mediated alignment during metal nanoparticle coalescence 
Fig. 1
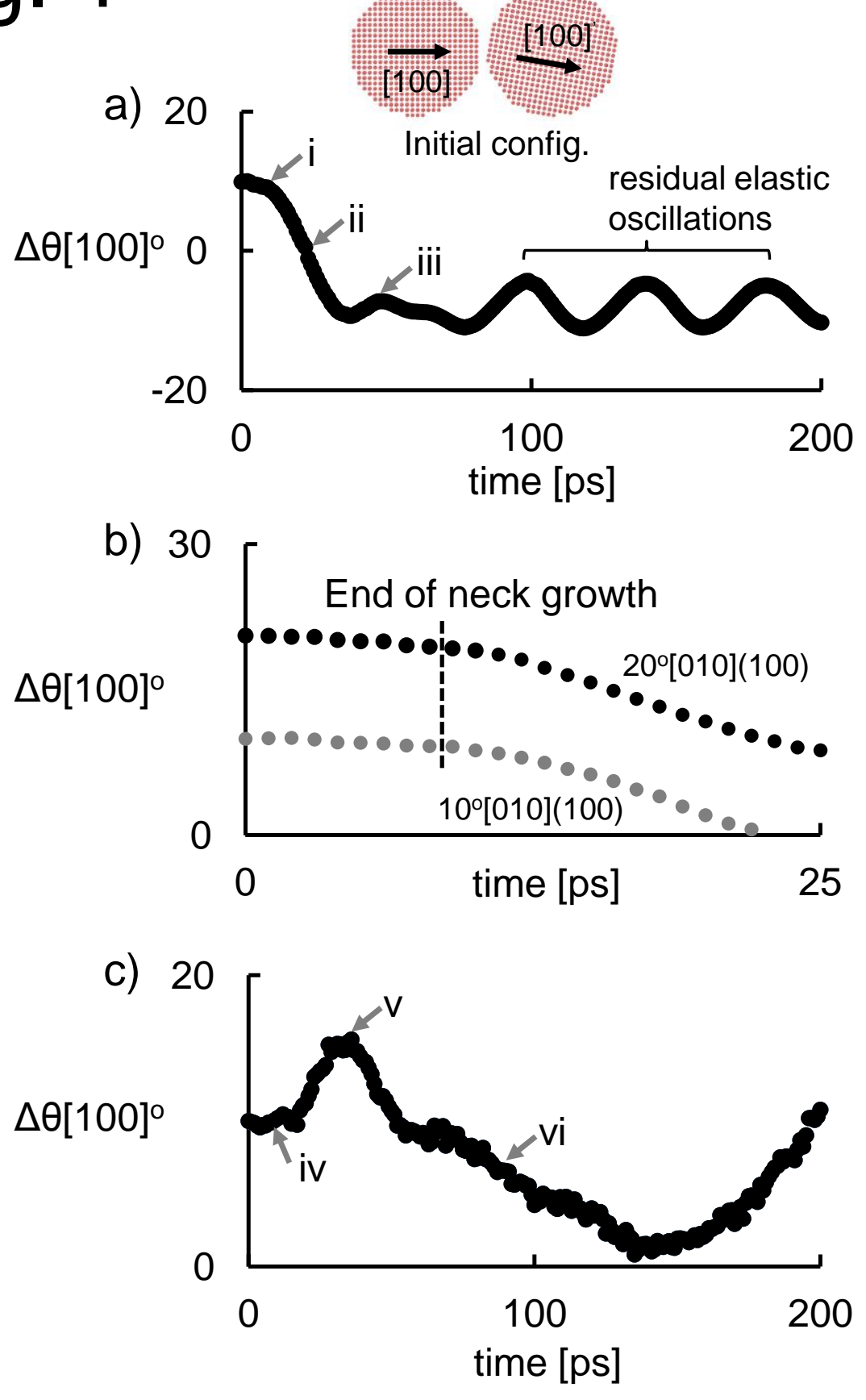

i) End of neck growth

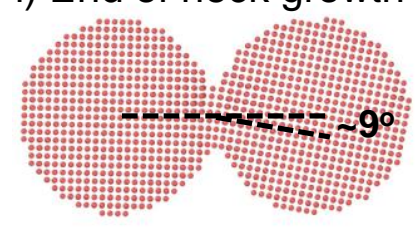

ii) Rotation

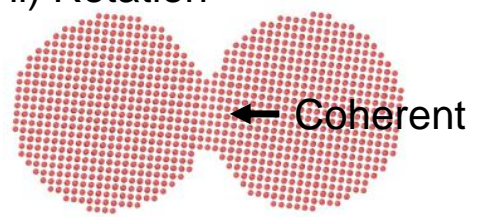

iii) Plastic deformation

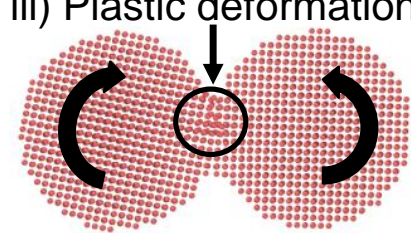

iv) End of neck growth

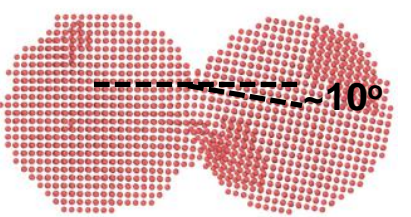

v) Slip in both particles

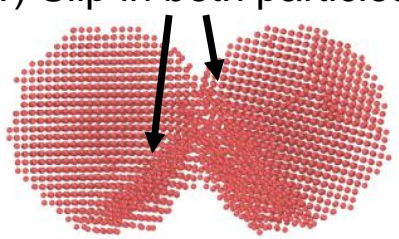

vi) Slip across GB

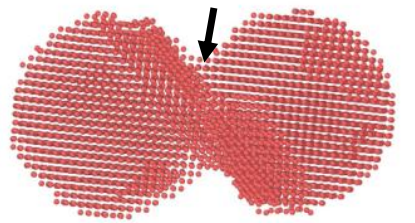

Fig.1. Rigid body rotations occurred after neck formation at $500 \mathrm{~K}$. Minimal rigid body rotations observed during attachment at $1100 \mathrm{~K}$. Plots of angular separation between [100] crystallographic axes $(\Delta \theta[100])$ in coalescing particles as a function of time shown in (a)-(c). Particles underwent extensive rotation after neck formation for $500 \mathrm{~K}$, $10^{\circ}[010](100)$ events (a). Atomic structures at different stages of attachment shown in (i)-(iii). Comparison of $\Delta \theta[100]$ vs. time for initial misorientations of $10^{\circ}[010](100)$ and $20^{\circ}[010](100)$ at $500 \mathrm{~K}$ in (b) shows that the angular velocity was nearly equal in the two cases. $\Delta \theta[100]$ plot for $1100 \mathrm{~K}, 10^{\circ}[010](100)$ simulation in (c) and corresponding structures (iv)(vi) show particles underwent minimal rotation during and after neck formation and slip on planes parallel to neck groove led to improved particle coalignment. 

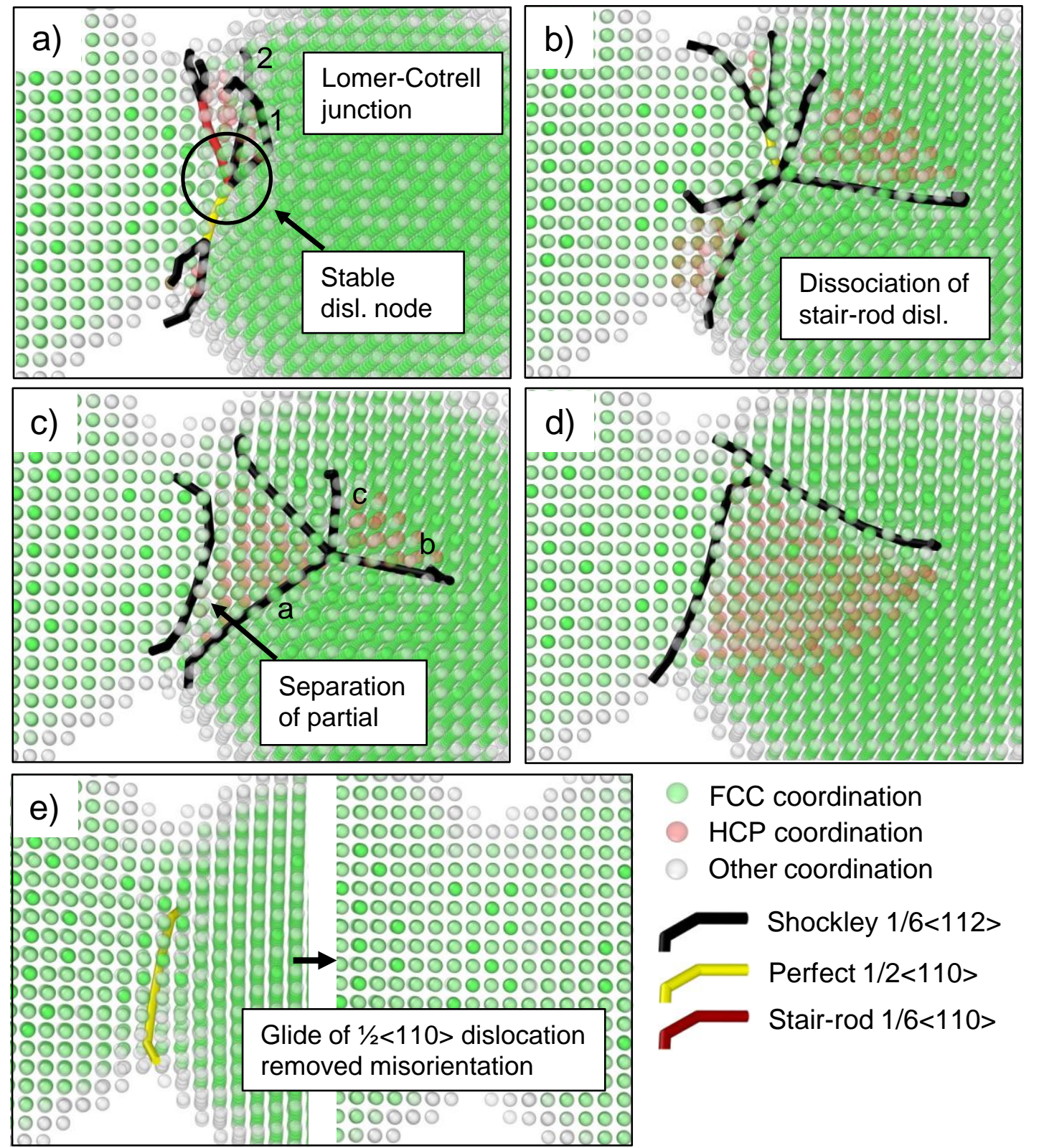

Fig. 2. At $500 \mathrm{~K}$, interfacial dislocations dissociated and glided to surface after imperfect attachment which led to improved co-alignment. Dislocation node in $45^{\circ}[100](100), 500 \mathrm{~K}$ simulation remained at the interface for $\sim 4 \mathrm{~ns}$ (a), preserving the misorientation. Node consisted of a Lomer-Cotrell lock (red dislocation and black dislocations labeled 1 and 2 in (a)) and a perfect dislocation (displayed in yellow). The red stair-rod dislocation dissociated (b) and a partial separated from the node (c). Three other partials (labeled a, b, and c) reacted (c), leaving two partials bounding a single stacking fault (d). These partials then reacted to form a perfect dislocation (e) which glided to the surface and left the particles aligned. 


\section{Fig. 3}
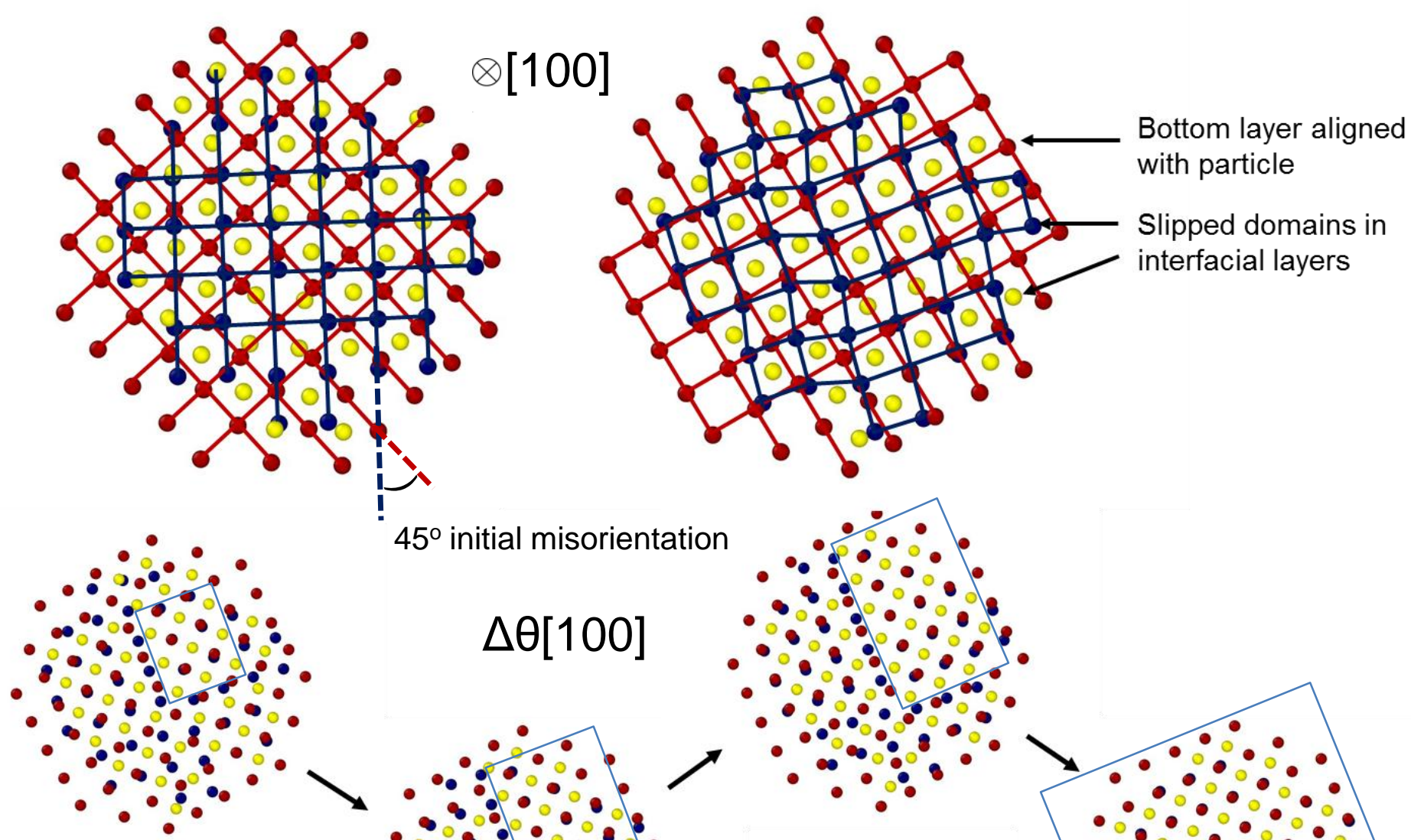

2 layer strain accommodation for low twist misorientation

$45^{\circ}$ initial misorientation
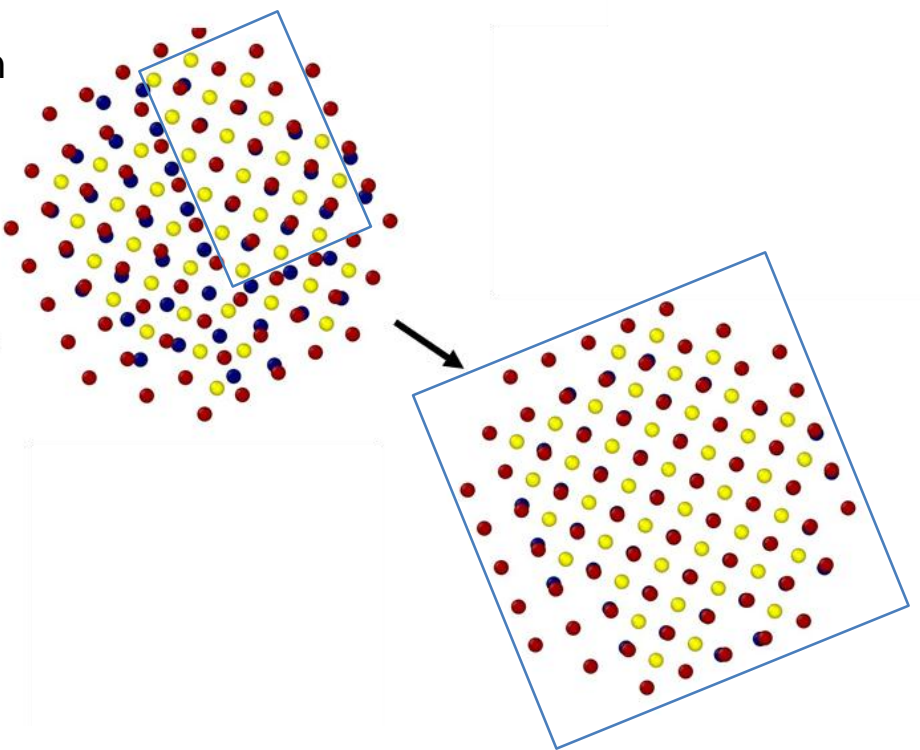

Fig. 3. Strain between particles with twist misorientation after neck formation accommodated by sheared interfacial layers. Three interfacial layers shown with their common [100] axis into the page. Red and yellow atoms initially belonged to one particle, blue atoms belonged to the other. Top left image shows snapshot of layers at the beginning of neck formation when misorientation was $45^{\circ}$. Top right image shows sheared domains within layers consisting of yellow and blue atoms. Degree of shearing reduced as misorientation decreased and number of coincident sites increased, as shown by blue rectangles in lower images. 


\section{Fig. 4}
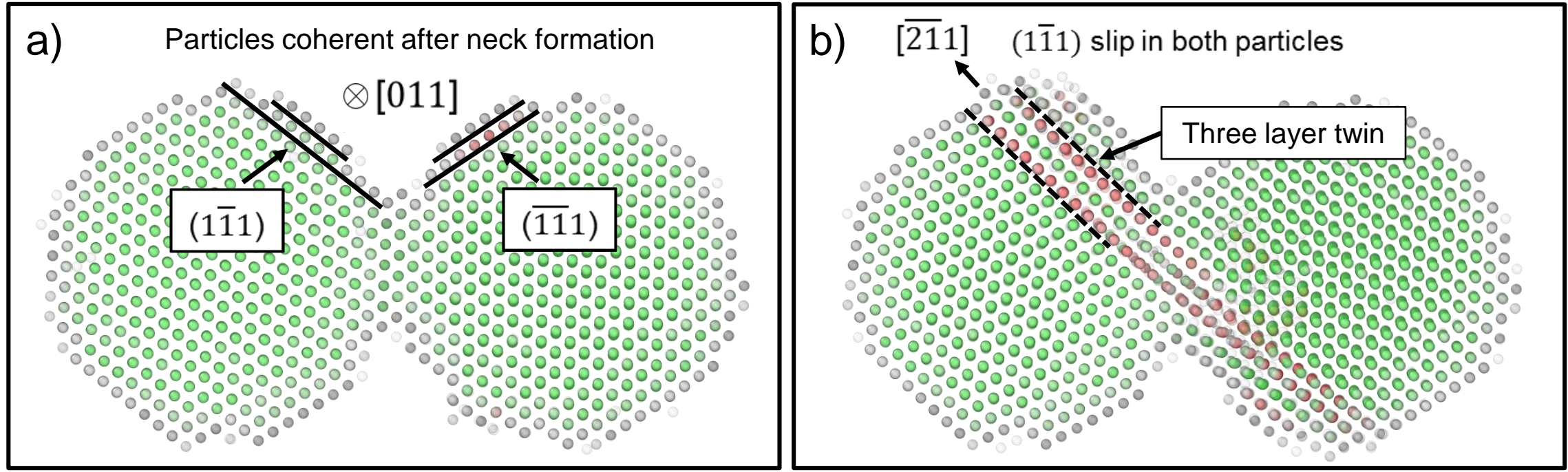

\section{C) Rotation due to dislocations at interface}
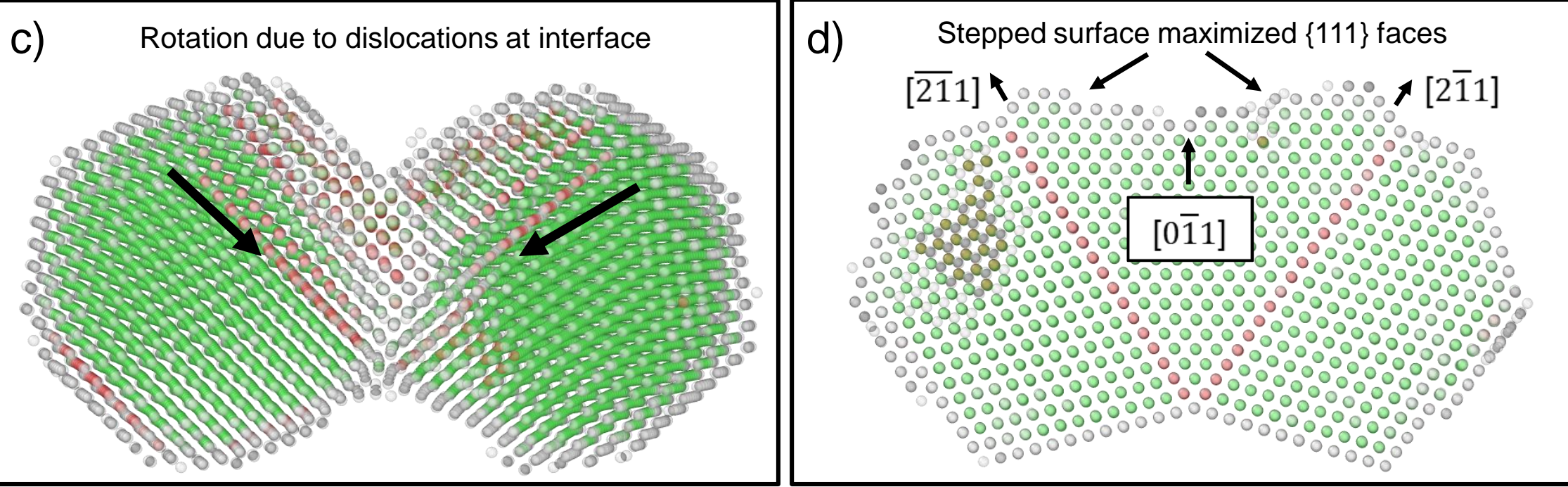

Fig.5. Deformation twinning during coalescence of initially aligned particles with common (100) faces at $1100 \mathrm{~K}$. Particles

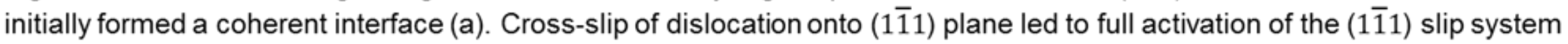
(b). (111) slip system then became active and particles rotated due to strain at the intersection of the two sets of $\{111\}$ slip planes (c). Two-fold, v-shaped twin formed due to deformation in both the [ $\overline{21} 1]$ and [2 $\overline{1} 1]$ directions (d). 

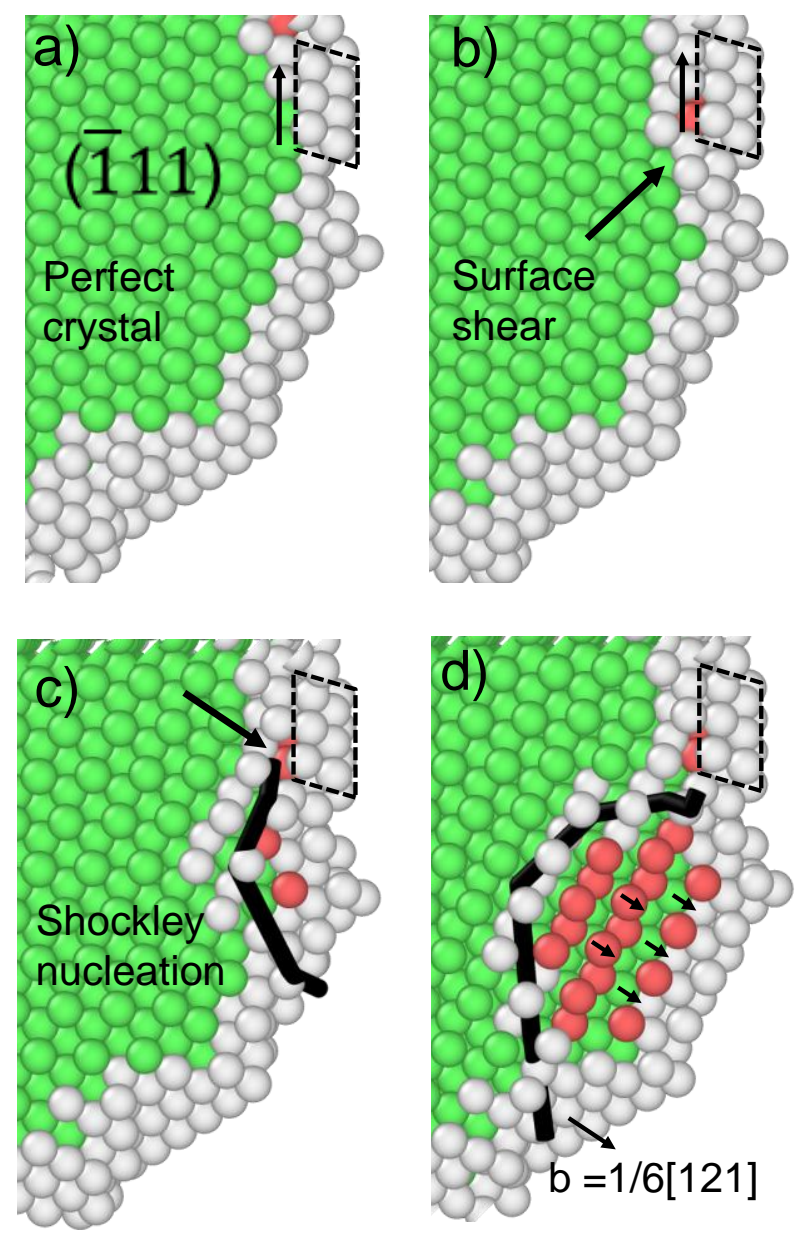

FCC coordination
HCP coordination
"Other" coordination

Fig. 5. Schockley partials nucleated from surface shearing events after coherent attachment during $1100 \mathrm{~K}, 0^{\circ}(100)$ simulation. Slip of surface atoms outlined by dotted line in ( $a$ and $\left.b\right)$ generated a stacking fault below the surface, evident from the red atom with hcp coordination in (b). Schockley partial glided on (111) plane and terminated on stacking fault under sheared surface layer (arrow in c). Partial then glided into interface as shown in (d). 
a)

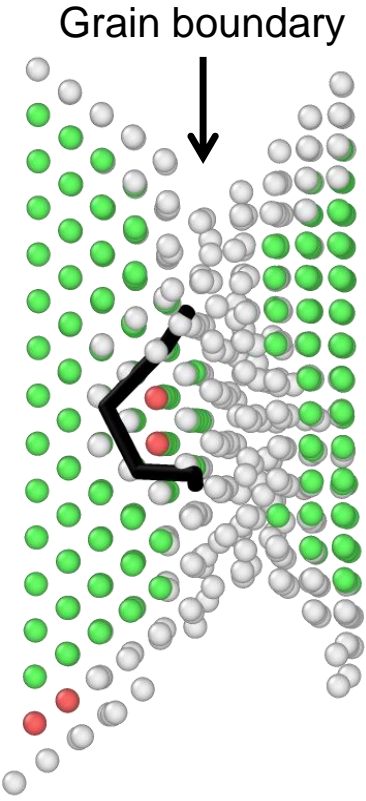

- FCC coordination

- HCP coordination

o "Other" coordination

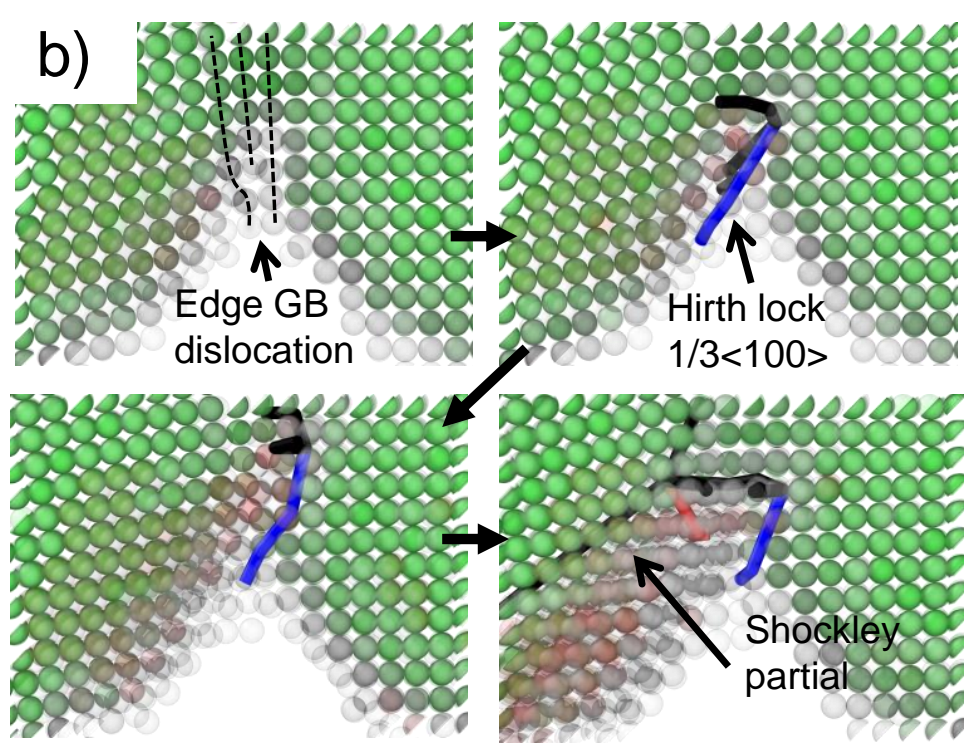

FCC coordination
HCP coordination
"Other" coordination

Fig. 6. Dislocation nucleation mechanisms prior to the activation of $\{111\}$ slip in $1100 \mathrm{~K}$ simulations differed for varied initial misorientations. Partials nucleated from disordered

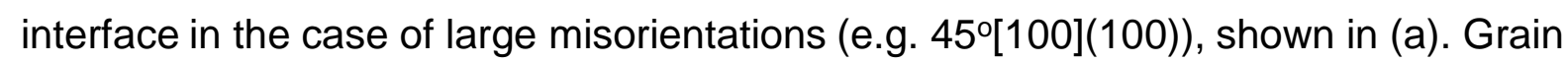
boundary dislocations dissociated and cross slipped in the case of low angle tilt misorientations (e.g. 10[100](100)), shown in (b). 


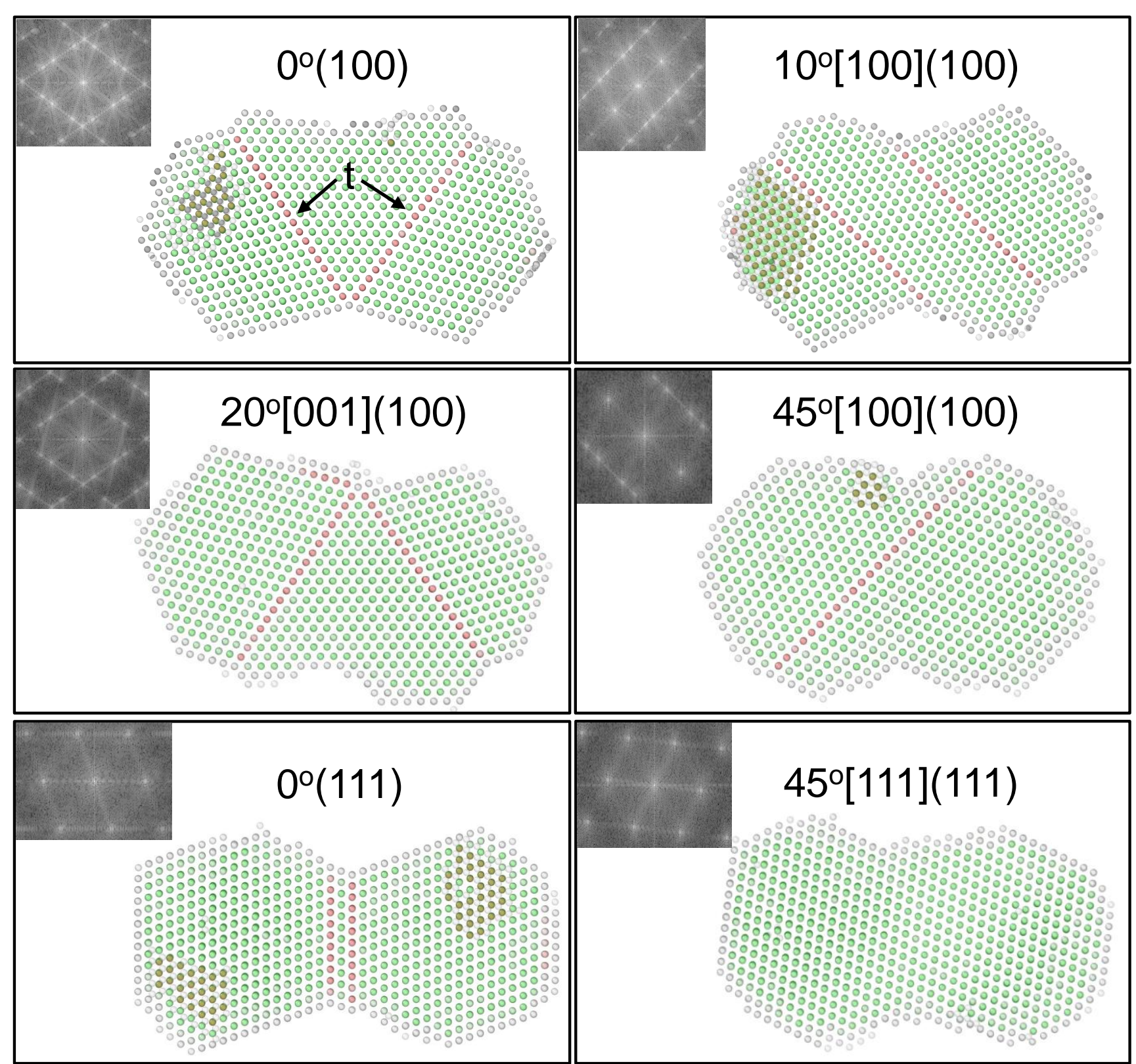

Fig. 7. All high temperature $(1100 \mathrm{~K})$ simulations led to twin formation except case in which particles had initial 45111](111) misorientation. Twinned structures labeled by initial misorientation and insets show FFT images of associated structures. Atoms along twin boundaries are colored red due to hcp coordination as indicated by t in top left panel. 


\section{Fig. 8}
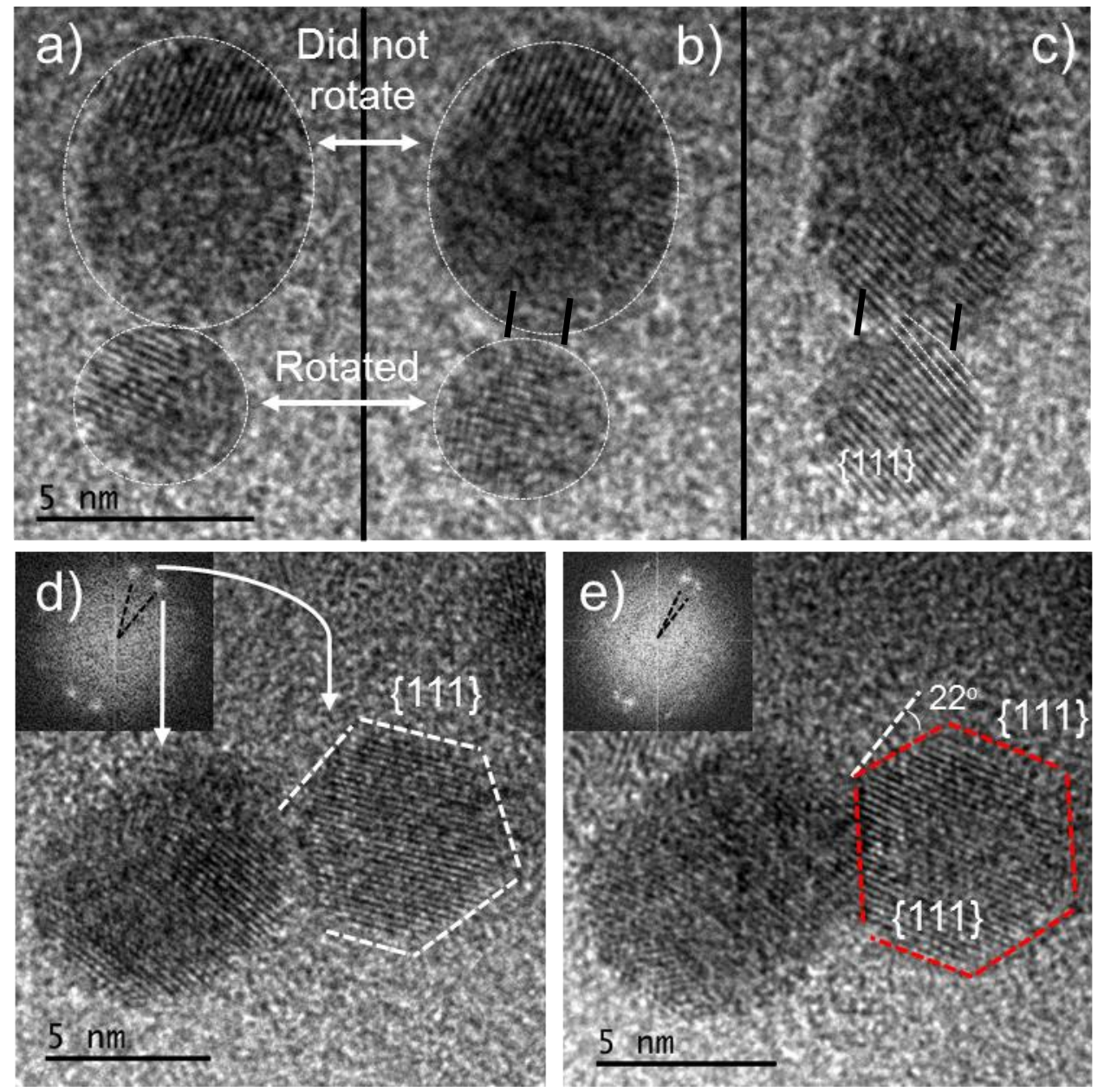

Fig. 8. Particles rotated after attachment (neck formation) during beam exposure experiments ( $T$ $\leq 315 \mathrm{~K}$ ). Lattice fringes show bottom particle rotated between frames (a) and (b) and both particles rotated between frames (b) and (c) which led to near alignment of $\{111\}$ planes indicated by dashed, white lines. Increase in neck width between frames (b) and (c) indicated by dark black lines. Right particle in (d) also underwent a $\sim 22^{\circ}$ rotation between frames (d) and (e) in a separate coalescence event after attachment as shown by the FFT insets and rotation of the surface facets, as indicated by the dashed lines. 

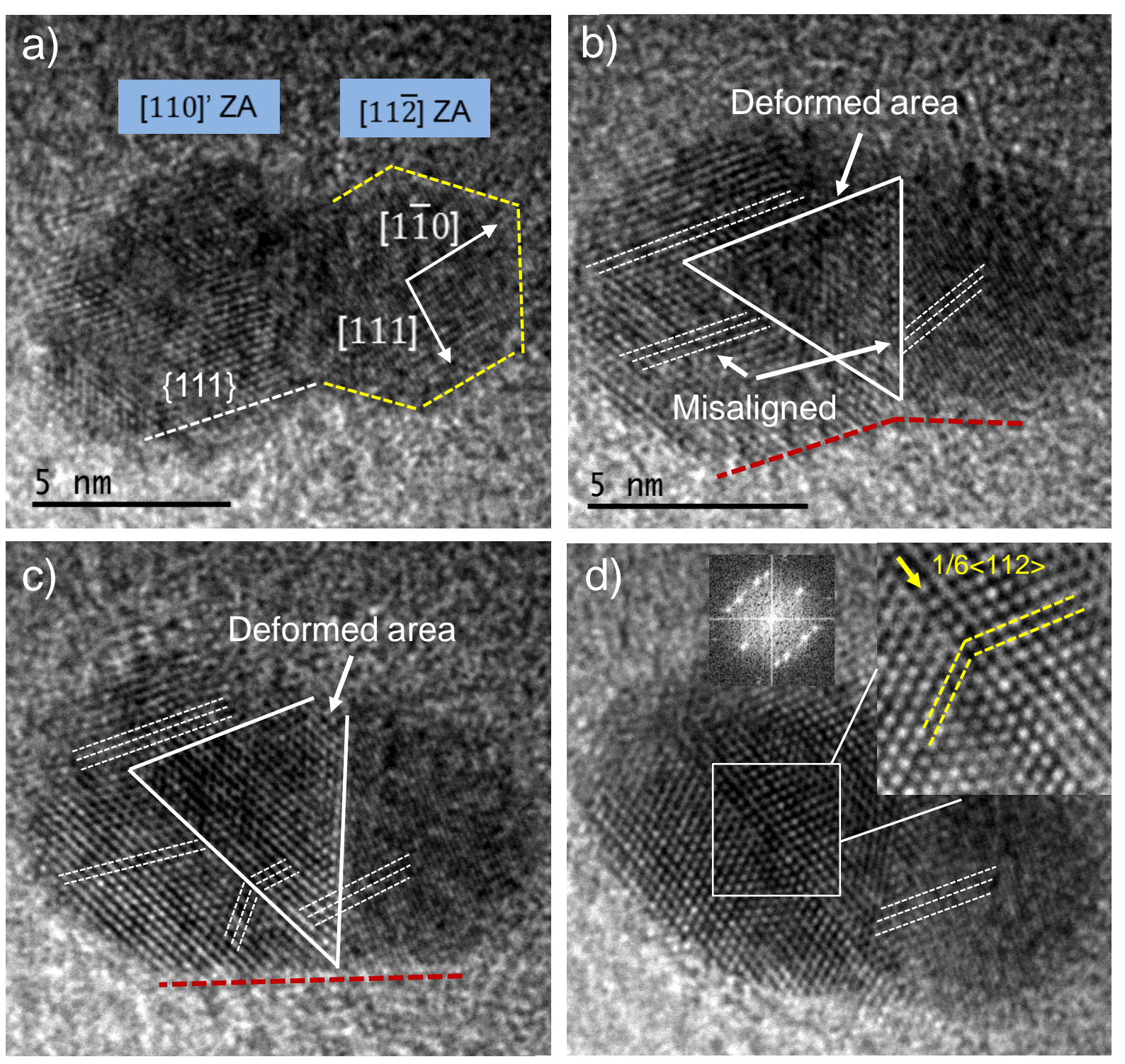

Fig. 9. Deformation twinning occurred after neck formation in coalesced particles under e-beam exposure $(\mathrm{T} \leq 315 \mathrm{~K})$. Zone axes of particles shown in (a). Atomic columns in triangular region in (b) were not visible, suggesting deformation occurred. Atomic columns in triangular region then became visible (c) and the $\{111\}$ planes in this region were parallel to the $\{111\}$ planes in the particle on the right. Twin boundary seen between the deformed and un-deformed regions (d), further emphasized by the selected area and FFT insets. Scale in (c) and (d) same as (b). 


\section{Fig. 10}
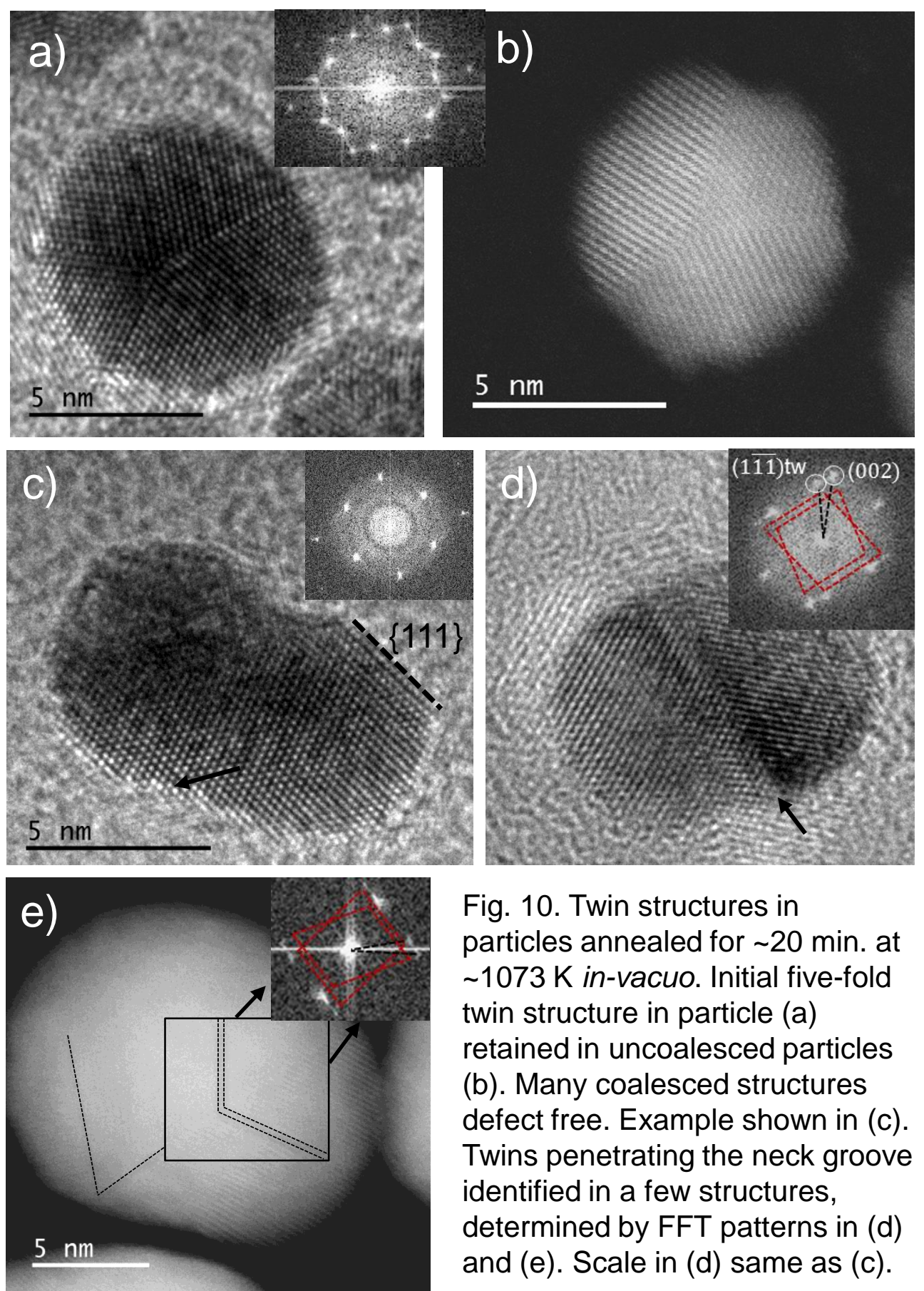

Fig. 10. Twin structures in particles annealed for $\sim 20 \mathrm{~min}$. at $\sim 1073 \mathrm{~K}$ in-vacuo. Initial five-fold twin structure in particle (a) retained in uncoalesced particles (b). Many coalesced structures defect free. Example shown in (c). Twins penetrating the neck groove identified in a few structures, determined by FFT patterns in (d) and (e). Scale in (d) same as (c). 
Classical model

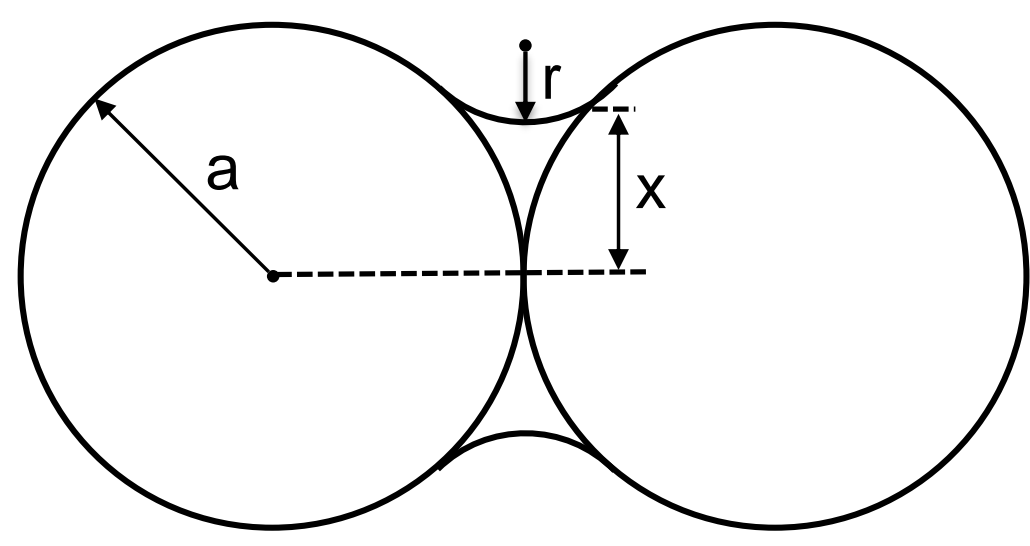

Faceted model

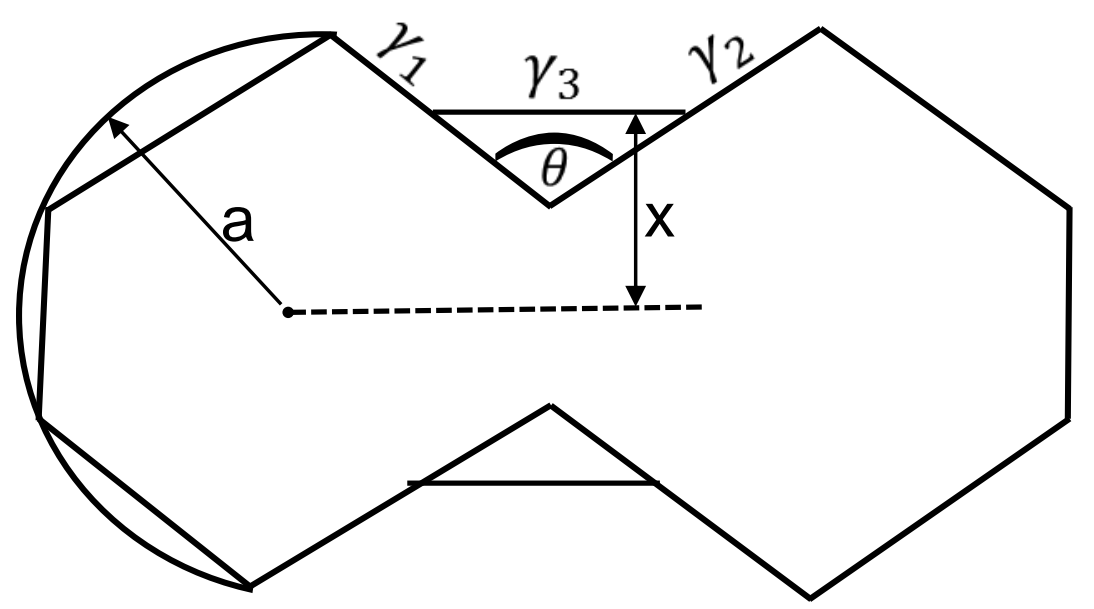

Fig. 11. Classical (top) and faceted (bottom) schematic representations of coalescence. Neck growth in classical model driven by chemical potential gradient (small radius of curvature, $r$ ) in neck groove. Neck growth by diffusion may not occur if particles are faceted due to stability of intersecting facets. 

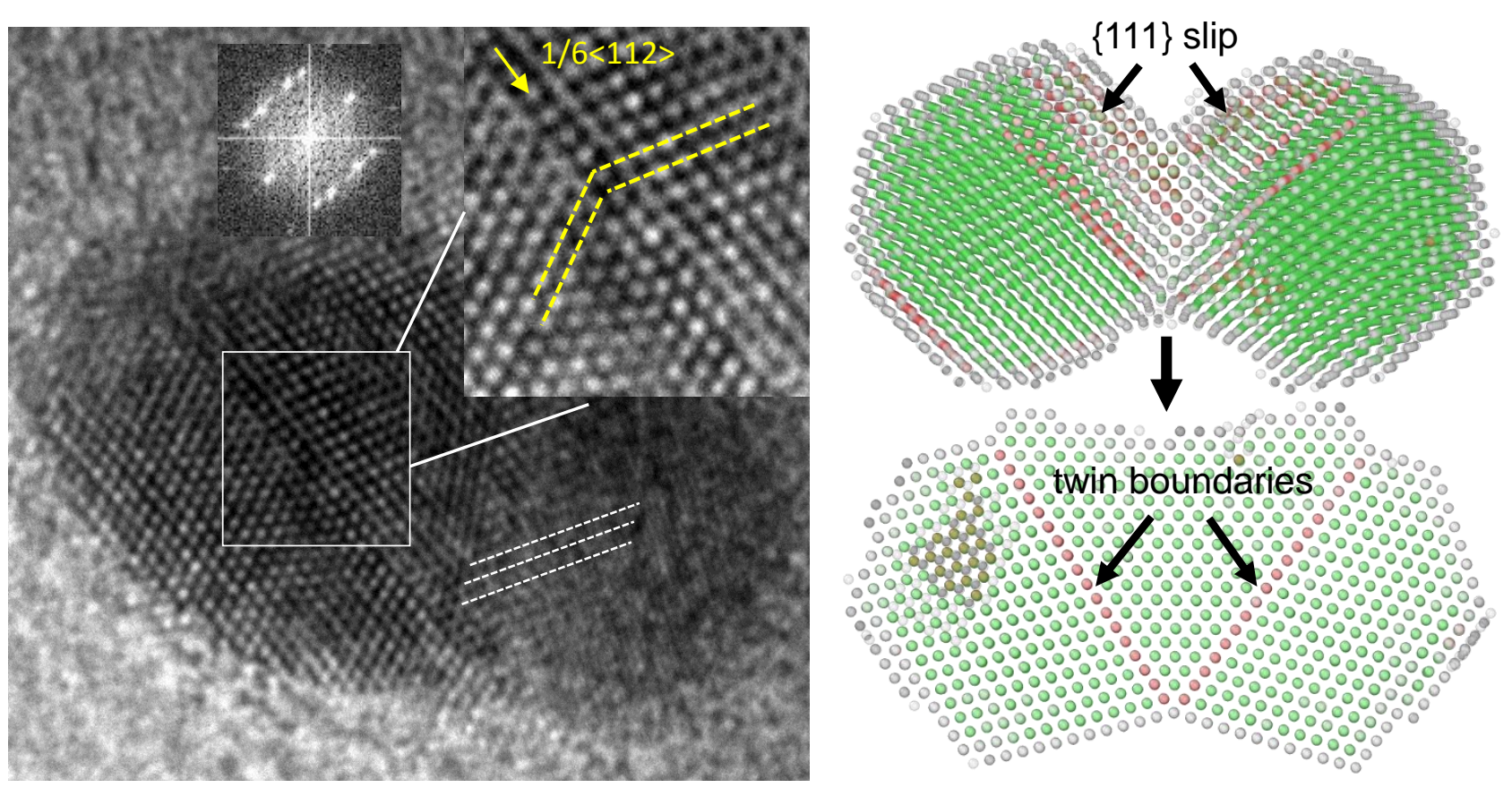